\title{
Eyelid ptosis and muscle weakness in a child with Kawasaki disease: a case report
}

\author{
Yao Lin, Lijun Wang, Aijie Li, Hongwei Zhang and Lin Shi
}

\begin{abstract}
Background: Kawasaki disease (KD) is an acute febrile vasculitis that often occurs in children under 5 years. Ptosis and muscle weakness associated with KD are rarely documented.

Case presentation: We present a case of KD with eyelid ptosis and muscle weakness in a 3-year-old boy. At admission, grade IV and grade III muscle strength were recorded for upper and lower limbs, respectively. Diminished patellar tendon reflex was noted. Laboratory evaluation showed hypokalemia with the serum potassium concentration of $2.62 \mathrm{mmol} / \mathrm{L}$. Intravenous immunoglobulin (IVIG) and aspirin were initiated immediately accompanied with methylprednisolone for adjunctive therapy. Potassium supplement was administered at the same time, which resulted in the correction of hypokalemia on the 2 nd day of admission but no improvement in ptosis and muscle weakness. Neostigmine testing, lumber puncture, electromyography, and cerebral and full spine MRI were performed, which, however, did not find evidence for neural and muscle diseases. On the 5th day, the fever was resolved. On the 6th day, eyelid ptosis disappeared. And on the 14th day, the muscle strength and muscle tension returned to normal, patellar tendon reflex could be drawn out normally, and the boy regained full ambulatory ability.
\end{abstract}

Conclusions: KD might affect the neural and muscular systems, and KD complicated with eyelid ptosis and muscle weakness is responsive to the standard anti-inflammatory treatment plus adjunctive corticosteroid therapy.

Keywords: Kawasaki disease, Ptosis, Muscle weakness, Myositis, Case report

\section{Article summary}

A 3-year-old boy with typical Kawasaki disease also presenting with eyelid ptosis and muscle weakness was treated with a standard anti-inflammatory regimen plus adjunctive therapy in acute stage and recovered completely.

\section{Background}

Kawasaki disease (KD) is an acute febrile vasculitis that often occurs in children under 5 years. Coronary artery lesions are the most common complications of $\mathrm{KD}$. Neural system complications are uncommon, which mainly include febrile convulsion, aseptic meningitis and

*Correspondence: shilin9789@126.com

Department of Pediatric Cardiology, Children's Hospital, Capital Institute of Pediatrics, No. 2 Yabao Road, Chaoyang District, Beijing 100020, China auditory nerve palsy [1]. Eyelid ptosis and muscle weakness, especially the simultaneous presence of these two conditions in acute phase of the disease, are rarely documented. In this article, we present a case of eyelid ptosis and muscle weakness secondary to KD.

\section{Case presentation}

A 3-year-old boy with fever for 5 days was admitted to our hospital. KD was diagnosed by typical symptoms including rashes, strawberry tongue, cervical lymphadenitis, conjunctivitis, and extremity edema. Besides these symptoms, the boy was noted to have eyelid ptosis (Fig. 1), muscle weakness with diminished patellar tendon reflex, nasal congestion and mastoid tenderness. The muscle strength of upper limbs was scaled as grade IV, and lower limbs grade III. Blood testing was performed, which revealed increased white blood 


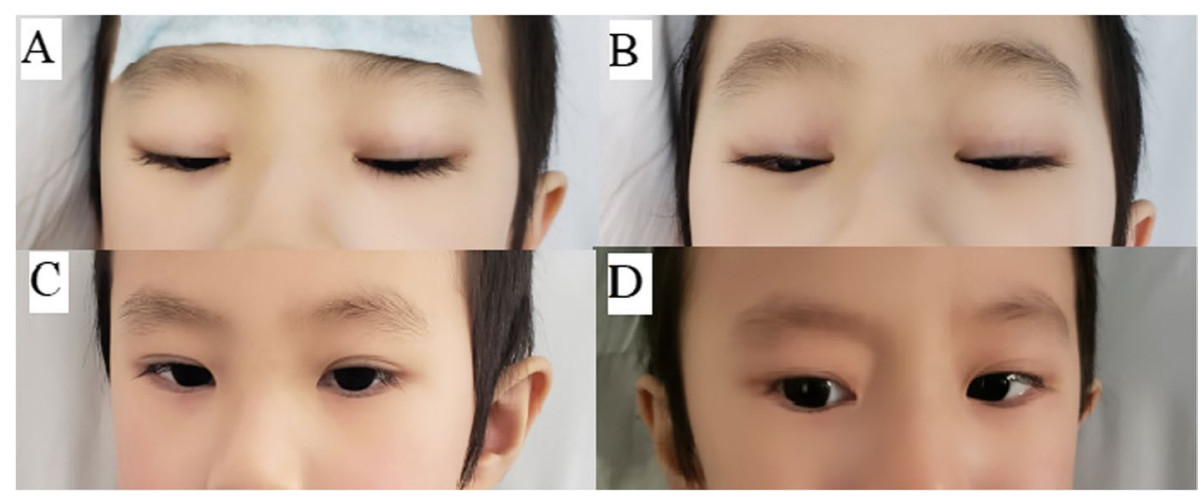

Fig. 1 The time course of eyelid ptosis in a 3-year-old boy with KD. A the 1st day of admission; $\mathbf{B}$ the 3rd day of admission; $\mathbf{C}$ the 6 th day of admission; and D: the 14th day of admission

Table 1 Blood testing results at admission

\begin{tabular}{ll}
\hline Parameter & Result \\
\hline White blood cell count & $22.48 \times 10^{9} / \mathrm{L}$ \\
Neutrophil ratio & $97 \%$ \\
C-reactive protein & $173.89 \mathrm{mg} / \mathrm{L}$ \\
Procalcitonin & $17.67 \mathrm{ng} / \mathrm{ml}$ \\
Erythrocyte sedimentation rate & $82 \mathrm{~mm} / \mathrm{h}$ \\
Alanine transaminase & $101.7 \mathrm{U} / \mathrm{L}$ \\
Aspertate aminotransferase & $42.1 \mathrm{U} / \mathrm{L}$ \\
Total bilirubin & $33.2 \mu \mathrm{mol} / \mathrm{L}$ \\
Direct bilirubin & $23.6 \mu \mathrm{mol} / \mathrm{L}$ \\
$\mathrm{K}^{+}$ & $2.62 \mathrm{mmol} / \mathrm{L}$ \\
Na & $128 \mathrm{mmol} / \mathrm{L}$ \\
D-Dimer & $2 \mathrm{mg} / \mathrm{LEU}$ \\
Serum albumin & $24.4 \mathrm{~g} / \mathrm{L}$ \\
Creatine kinase & $62 \mathrm{U} / \mathrm{L}$ \\
Blood gas analysis & $\mathrm{metabolic}$ acidosis \\
\hline
\end{tabular}

cell counts, elevated C-reactive protein and transaminases, and decreased $\mathrm{K}^{+}$(Table 1). Echocardiography showed normal bilateral coronary artery but minor regurgitation in the mitral and tricuspid valves. Cranial CT suggested otitis media and mastoiditis. As COVID19-associated multisystem inflammatory syndrome overlaps with $\mathrm{KD}$, the patient underwent testing for COVID-19. Both RT-PCR and antibody measurement were negative. Intravenous immunoglobulin (IVIG) with the dosage of $2 \mathrm{~g} / \mathrm{kg}$ and aspirin with the dosage of $30 \mathrm{mg} / \mathrm{kg} / \mathrm{d}$ were given immediately. Furthermore, according to the Kobayashi risk stratification [2], methylprednisolone infusion was initiated $(2.8 \mathrm{mg} / \mathrm{kg} / \mathrm{d}$, administered every $8 \mathrm{~h}$ ) for adjunctive anti-inflammatory therapy. Other therapies were administered at the same time, including latamoxef recommended by an otorhinolaryngologist for otitis media and mastoiditis, human albumin infusion, potassium supplement, and glutathione (reduced) and glycyrrhizin for hepatoprotection.

On the 2nd day of admission, the hypokalemia and hypoalbuminemia were corrected with the serum concentration of $3.89 \mathrm{mmol} / \mathrm{L}$ and $31.7 \mathrm{~g} / \mathrm{L}$ respectively. However, fever, eyelid ptosis and muscle weakness were not improved. Family history of disease with similar symptoms was denied and toxin exposure was excluded. Neostigmine testing, lumber puncture, and cerebral and full spine MRI were performed, which, however, did not show evidence for neural and muscular diseases such as myasthenia gravis, Guillain-Barre syndrome and meningitis. On the 5 th day of admission, the fever was resolved, and the lower limb muscle strength was recovered gradually to grade IV with a weak patellar tendon reflex elicited. Nasal congestion and mastoid tenderness were improved gradually. On the 6th day of admission, eyelid ptosis disappeared; ophthalmoscopy, electromyography and metabolism disease screening showed normal results; white blood cell count and C-reactive protein became normal; and aspirin was reduced to $3 \mathrm{mg} / \mathrm{kg}$ daily. On the 8th day of admission, 5 days after the initial KD rashes disappeared, the boy started to develop new itching rashes over his trunk, and the blood routine showed elevated eosinophil counts. Drug allergy was suspected, and aspirin and latamoxef were replaced by clopidogrel and ertapenem respectively. Cetirizine was administered for anti-allergy therapy. The rashes faded 3 days later. Methylprednisolone dosage was reduced to $1 \mathrm{mg} / \mathrm{kg} / \mathrm{d}$ and tapered in 4 weeks (completed in outpatient clinic). On the 14th day of admission, the muscle strength and muscle tension returned to normal completely, patellar tendon reflex could be drawn out normally, and the boy regained full ambulatory ability. Laboratory evaluation 
showed normal hepatic and renal function, stable electrolytes, and normal D-Dimer. Echocardiography showed normal size and function of the heart without any regurgitation and normal inner diameter of bilateral coronary artery. Hearing screening was normal. Whole-exome sequencing did to identify genetic abnormalities. The patient was discharged after 20 days of hospitalization. At the 1-month follow-up after discharge, the boy was healthy without muscle weakness and eyelid ptosis recurrence.

\section{Discussion and conclusion}

Eyelid ptosis or muscle weakness associated with KD is uncommon. We present here a case of concurrent eyelid ptosis and muscle weakness in the acute stage of KD, an even rarer entity. A singular neurological symptom associated with KD has been described in several case reports [3-10]. Simultaneous presence of ptosis and limb muscle weakness in a patient with KD is novel. Koutras reported that an 18-month-old girl with KD had proximal muscle weakness, dysphonia and dysphagia on the 8th day of illness [3]. Although laboratory examination showed normal creatine kinase levels, electromyography revealed myositis. Myositis associated with KD eventually diagnosed by electromyography was also described in 2 other cases $[4,5]$. Additionally, 4 cases of myositis concurrent with KD were confirmed by muscle biopsy [4-7]. We tried to identify the potential cause for ptosis and muscle weakness in our case. However, no elevated serum creatine kinase was detected and no muscle damages were observed; results of neurological exams were normal and genetic cause was also excluded. It is unlikely that hypokalemia caused ptosis and muscle weakness in our patient as correction of hypokalemia did not result in improvement of ptosis and muscle weakness.

Despite the confirmatory diagnosis of myositis in KD reported previously, the mechanism of myositis is unresolved. We speculated that vasculitis around the affected muscle and nerve might be the culprit, but why only palpebralis and limb muscles were affected remains unknown. Further study is warranted, which, however, is challenging due to the rarity of the condition.

Different treatment methods have been applied for ptosis secondary to KD. Zhao et al. treated a 5-year-old girl with typical $\mathrm{KD}$, and the patient developed ptosis 3 days after IVIG administration. After a series of examinations, oculomotor nerve palsy was diagnosed. The child was given aspirin and prednisone, and recovered 4 weeks later with symptoms of ptosis beginning to relieve 3 weeks after the initiation of aspirin and prednisone [11]. Thapa et al. reported that two boys with KD who developed right-side oculomotor nerve palsy that was manifested by ipsilateral ptosis and medial rectus palsy recovered 5 days after
IVIG treatment [12]. Another study showed that a patient with KD complicated with orbital cellulitis had eyelid movement restriction that regressed 3days after aspirin administration [13]. Hameed et al. reported that a 3-yearold boy developed bilateral ptosis on day 21 ( 5 days after IVIG administration), which resolved on day 25 without any other special therapies [14]. In the present case, the patient received IVIG, aspirin and methylprednisolone for the management of KD complicated with eyelid ptosis and muscle weakness, and the outcome is satisfactory. These data indicate that in addition to the standard anti-inflammatory treatment, adjunctive therapy might be required for the effective management of eyelid ptosis and muscle weakness associated with $\mathrm{KD}$, which is helpful for clinicians working in the field.

In conclusion, KD might affect the neural and muscular systems, and KD complicated with eyelid ptosis and muscle weakness is responsive to the standard anti-inflammatory treatment plus adjunctive corticosteroid therapy.

\section{Abbreviations \\ KD: Kawasaki disease; IVIG: Intravenous immunoglobulin.}

\section{Acknowledgements}

We would like to thank the parents of the patient for providing consent to the publication of this case.

\section{Authors' contributions}

Dr. YL and LS conceptualized and designed the study, drafted the initial manuscript, critically reviewed and revised the manuscript. Dr. LW collected data, reviewed and revised the manuscript. Dr. HZ and AL coordinated and supervised data collection, reviewed and revised the manuscript. All authors have read and approved the final manuscript as submitted and agreed to be accountable for all aspects of the work.

\section{Funding}

None.

Availability of data and materials

The data of this case are available from the corresponding author on reasonable request.

\section{Declarations}

\section{Ethics approval and consent to participate}

This case report was approved by Medical Research Review Board of Children's Hospital, Capital Institute of Pediatrics, Beijing, China (approval number: SHERLL2021033). The written informed consent was obtained from the parents of the patient.

\section{Consent for publication}

The written consent for publication was obtained from the parents of the patient.

\section{Competing interests}

All authors declare no competing interests.

Received: 14 July 2021 Accepted: 28 October 2021

Published online: 27 November 2021 


\section{References}

1. McCrindle BW, Rowley AH, Newburger JW, et al. Diagnosis, treatment, and long-term Management of Kawasaki Disease: a scientific statement for health professionals from the American Heart Association. Circulation. 2017;135(17):e927-99.

2. Kobayashi T, Inoue Y, Otani T, et al. Risk stratification in the decision to include prednisolone with intravenous immunoglobulin in primary therapy of Kawasaki disease. Pediatr Infect Dis J. 2009;28(6):498-502.

3. Koutras A. Myositis with Kawasaki's disease. Am J Dis Child. 1982;136(1):78-9

4. Sugie H, Sugie Y, Ichimura M, Mizuno Y, Nishida M, Igarashi Y. A case of polymyositis associated with Kawasaki disease. Brain Dev. 1985;7(5):513-5

5. Gama C, Breeden K, Miller R. Myositis in Kawasaki disease. Pediatr Neurol. 1990;6(2):135-6.

6. Lin H, Burton EM, Felz MW. Orbital myositis due to Kawasaki's disease. Pediatr Radiol. 1999;29(8):634-6.

7. Lee EY, Oh JY, Chong CY, Choo JT, Mahadev A, Tan NW. A Case of Atypical Kawasaki Disease with Myositis. Glob Pediatr Health. 2015:2:2333794X15599649.

8. Agarwal S, Gupta A, Suri D, Rawat A, Singh S. Proximal muscle weakness in a child with Kawasaki disease. Indian J Pediatr. 2015;82(9):866.

9. Vigil-Vazquez S, Butragueno-Laiseca L, Lopez-Gonzalez J, Garcia-San Prudencio M, Rincon-Lopez E. A case of Kawasaki disease presenting as severe myositis. Indian J Pediatr. 2019;86(11):1066-7.
10. Anjani G, Johnson N, Navid A, Saka R, Jindal AK, Singh S. Kawasaki disease malingering as juvenile dermatomyositis: case-based review: myositis in Kawasaki disease. Rheumatol Int. 2021. https://doi.org/10.1007/ s00296-021-04826-2.

11. Zhao SH, Wang R, Ma LQ, Yang Y. Ptosis of both palpebra superiors caused by Kawasaki disease in a child. Zhongguo Dang Dai Er Ke Za Zhi. 2007;9(1):83.

12. Thapa R, Mallick D, Biswas B, Chakrabartty S. Transient unilateral oculomotor palsy and severe headache in childhood Kawasaki disease. Rheumatol Int. 2011;31(1):97-9.

13. Cerman E, Eraslan M, Turhan SA, Usta SA, Akalin F. Orbital cellulitis presenting as a first sign of incomplete Kawasaki disease. Case Rep Ophthalmol. 2013;4(3):294-8.

14. Hameed A, Alshara H, Schleussinger T. Ptosis as a complication of Kawasaki disease. BMJ Case Rep. 2017;2017. https://doi.org/10.1136/ bcr-2017-219687.

\section{Publisher's Note}

Springer Nature remains neutral with regard to jurisdictional claims in published maps and institutional affiliations.
Ready to submit your research? Choose BMC and benefit from:

- fast, convenient online submission

- thorough peer review by experienced researchers in your field

- rapid publication on acceptance

- support for research data, including large and complex data types

- gold Open Access which fosters wider collaboration and increased citations

- maximum visibility for your research: over $100 \mathrm{M}$ website views per year

At $\mathrm{BMC}$, research is always in progress.

Learn more biomedcentral.com/submissions 